\title{
Recent cadmium exposure among male partners may affect oocyte fertilization during in vitro fertilization (IVF)
}

\author{
Keewan Kim • Victor Y. Fujimoto • Patrick J. Parsons • \\ Amy J. Steuerwald • Richard W. Browne • \\ Michael S. Bloom
}

Received: 15 January 2010 / Accepted: 11 May 2010 /Published online: 27 May 2010

(C) The Author(s) 2010. This article is published with open access at Springerlink.com

\begin{abstract}
Purpose We recently reported evidence suggesting associations between urine cadmium concentrations, reflecting long-term exposure, measured in 25 female patients (relative risk $=1.41, P=0.412$ ) and 15 of their male partners (relative
\end{abstract}

Capsule Adjusted for long-term exposure, recent cadmium (Cd) exposure among the male partners of female in vitro fertilization patients may be inversely associated with oocyte fertilization.

\footnotetext{
K. Kim

Department of Epidemiology, University at Albany,

State University of New York,

Rensselaer, NY, USA

P. J. Parsons $\cdot$ A. J. Steuerwald $\cdot$ M. S. Bloom Department of Environmental Health Sciences, University at Albany, State University of New York, Rensselaer, NY, USA

\section{Y. Fujimoto}

Department of Obstetrics, Gynecology,

and Reproductive Sciences,

University of California at San Francisco,

San Francisco, CA, USA

P. J. Parsons $\cdot$ A. J. Steuerwald

The Laboratory of Inorganic and Nuclear Chemistry,

Wadsworth Center, New York State Department of Health,

Albany, NY, USA

R. W. Browne

Department of Biotechnical and Clinical Laboratory Sciences, University at Buffalo, State University of New York,

Buffalo, NY, USA

M. S. Bloom $(\bowtie)$

Department of Environmental Health Sciences,

School of Public Health,

Rm. \#153, One University Place,

Rensselaer, NY 12144, USA

e-mail:mbloom@uamail.albany.edu
}

risk $=0.19, P=0.097)$ and oocyte fertilization in vitro. Blood cadmium concentrations reflect more recent exposure.

Methods We here incorporate those measures into our prior data set and employ multivariable log-binomial regression models to generate hypotheses concerning the relative effects of long-term and recent cadmium exposure on oocyte fertilization in vitro.

Results No association is indicated for blood cadmium from women and oocyte fertilization, adjusted for urine cadmium and creatinine, blood lead and mercury, age, race/ ethnicity and cigarette smoking (relative risk $=0.88, P=$ 0.828 ). However, we suggest an inverse adjusted association between blood cadmium from men and oocyte fertilization (relative risk $=0.66, P=0.143$ ).

Conclusions These results suggest that consideration of long-term and recent exposures are both important for assessing the effect of partner cadmium levels on oocyte fertilization in vitro.

Keywords Cadmium (Cd) - Oocyte fertilization . In vitro fertilization (IVF) - Assisted reproductive technologies (ART)

\section{Introduction}

We recently reported data suggesting associations between long-term cadmium $(\mathrm{Cd})$ exposure and oocyte fertilization during in-vitro fertilization (IVF) treatment [1]. These results indicated the possibility of a positive association between concentrations of urine $\mathrm{Cd}$ and oocyte fertilization in female IVF patients (adjusted relative risk $(\mathrm{aRR})=1.41$ ). These prior results also suggest the possibility of an inverse association between urine $\mathrm{Cd}$ in male partners of IVF patients and oocyte fertilization $(\mathrm{aRR}=0.19)$. 
Urine $\mathrm{Cd}$ is generally recognized as a marker of longterm exposure, reflecting deposition in the kidney over time, and likely resulting from dietary sources in our study population. In this study, we expand our previous data set to incorporate blood $\mathrm{Cd}$ concentrations which reflect recent exposure, on the order of days to weeks [2]. Following exposure, a small proportion of $\mathrm{Cd}$ concentrates in the blood, primarily bound to erythrocytes, as well as to plasma albumin and metallothionein and reflects exposure during the preceding 2-3 months [3]. The aim of this preliminary study is to generate hypotheses concerning the relative contribution, if any, of recent $\mathrm{Cd}$ exposure to the effects suggested for long-term $\mathrm{Cd}$ exposure in our prior study.

\section{Methods}

Fifty-eight female patients and 36 male partners undergoing IVF treatment at the UCSF Center for Reproductive Health were recruited to the Study of Metals and Assisted Reproductive Technologies (SMART) between September 1st, 2007 and August 31st, 2008. Sample selection and clinical protocols were previously described in detail [1]. Female patients underwent gonadotropin-induced ovarian stimulation per clinic protocols. When at least two follicles exceeded $17 \mathrm{~mm}$ in diameter, human chorionic gonadotropin was administered and oocytes were retrieved $36 \mathrm{~h}$ later. Between two and 39 mature oocytes were collected from each woman. Collected oocytes were fertilized by conventional insemination or by intracytoplasmic sperm injection using sperm from male partners or a donor. Approximately $16-18 \mathrm{~h}$ following insemination, zygotes were identified by the appearance of two pronuclei. A single whole blood and a single urine specimen were obtained concurrently from each female patient at the time of oocyte retrieval, and from each male partner, when available, on the same day. Blood specimens were obtained from 50 female patients and 33 male partners. Informed consent was obtained from all study participants and the study protocol was approved by the Institutional Review Board of the University of California at San Francisco.

Blood and urine specimens were analyzed for Cd using methods validated for a Perkin Elmer Sciex ELAN DRC II inductively coupled plasma-mass spectrometer (PerkinElmer Life and Analytical Sciences, Shelton, CT) with dynamic reaction cell (DRC-ICP-MS) technology. For each study participant, $1.8 \mathrm{~mL}$ cryovials, containing whole blood or urine, were shipped on dry ice to the Wadsworth Center's Laboratory of Inorganic and Nuclear Chemistry at the New York State (NYS) Department of Health (Albany, NY) for analysis. The Wadsworth Center is certified under NYS, CLIA and OSHA regulations and successfully participates in proficiency testing (PT) pro- grams for blood and urine Cd operated by the NYS Department of Health, the Institut National de Santé Publique du Québec, Le Centre de Toxicologie du Québec, the University of Surrey, UK, as well as in the German External Quality Assessment Scheme, operated by the Institute and Outpatient Clinic for Occupational, Social and Environmental Medicine of the Friedrich-Alexander University, Erlangen-Nuremberg, Germany.

The analytic procedure has been previously described in detail [4] as have the details for the urine $\mathrm{Cd}$, blood hg and blood $\mathrm{Pb}$ analyses [1]. The analyses were carried out under Class 100 Clean Room conditions. A minimum of six Cd standards traceable to the National Institute of Standards and Technology (NIST) were used for calibration. Blood and urine specimens were analyzed for $\mathrm{Cd}$ by monitoring $\mathrm{m} / \mathrm{z}=114$. Four blood or urine levels of internal quality control (QC) materials were analyzed during each run. Typical coefficients of variation for these assays range from $3 \%$ to $6 \%$ for blood $\mathrm{Cd}$ and $1 \%$ to $3 \%$ for urine $\mathrm{Cd}$. Method accuracy is periodically assessed by analyzing NIST Standard Reference Material 966 (Toxic Metals in Bovine Blood), and NIST SRM 2670a (Toxic Elements in Urine). Method performance for blood and urine $\mathrm{Cd}$ is also assessed via participation in the four external quality assessment schemes previously mentioned. The limit of detection (LOD), defined as three times the standard deviation of concentrations measured in matrix blanks for 10 independent analyses, is $0.2 \mu \mathrm{g} / \mathrm{L}$ for blood and $0.02 \mu \mathrm{g} / \mathrm{L}$ for urine. Values were reported for research purposes without regard for the LOD to preclude the introduction of bias into statistical models [5].

Statistical significance was defined as $P<0.10$ for a twotailed test consistent with the hypothesis generation nature of this study. Non-parametric tests were employed to assess bivariate associations due to lack of normality. Multiple log-binomial regression was used to assess associations between oocyte fertilization (no/yes) and the concentrations of blood and urine $\mathrm{Cd}$ with adjustment for urine creatinine, blood $\mathrm{Hg}$, blood $\mathrm{Pb}$, age, race/ethnicity and cigarette smoking. These covariates were selected a priori for inclusion in multiple log-binomial regression models using literature review followed by incorporation into directed acyclic graphs (DAGs). Directed acyclic graphs employ causal graphing theory to identify a minimally sufficient set of variables with which to control confounding under a postulated causal pathway [6]. Clinical variables, including semen quality, ICSI vs. conventional insemination, and other treatment related factors represent intermediate variables in the causal pathway between exposure (i.e., metals concentrations) and endpoint (i.e., oocyte fertilizations). Thus, these variables were excluded from multivariable analysis to preclude the introduction of bias that would result from their incorporation [7]. Generalized 
estimating equations (GEE) were used to generate robust standard errors. The Quasi-likelihood information criterion (QIC) was used to assess model fit given the non-likelihood based nature of GEE; a smaller QIC value indicates improved model fit [8]. SAS v.9.2 (SAS Institute, Cary, NC) was used for all statistical analyses.

\section{Results}

Table 1 shows the distribution of blood $\mathrm{Cd}$ concentrations measured on the day of oocyte collection in female patients and their male partners; $68 \%$ and $42.4 \%$ of female and male values were above the LOD respectively. Concentrations of blood and urine $\mathrm{Cd}$ are correlated in female patients $(r=0.47$, $P=0.0008)$ but not in male partners $(r=0.17, P=0.350)$. A larger proportion of each woman's oocytes were fertilized by ICSI (median $=0.76, n=37$ ) than by conventional insemination (median=0.56, $n=21$ ). However, no significant difference is detected by insemination protocol for concentrations of metals measured in women $(P>0.219)$ or men $(P>0.235)$.

The log-binomial regression of oocyte fertilization on urine and blood $\mathrm{Cd}$ measured in 25 female patients is presented in Table 2. As reported previously, Model 1 for female patients including urine $\mathrm{Cd}$ as the sole predictor of interest suggests a $41 \%$ increase in the probability for oocyte fertilization per $\mu \mathrm{g} / \mathrm{L}$ increase in urine $\mathrm{Cd}$. In Model 2, the addition of blood $\mathrm{Cd}$ concentrations to Model 1 has little impact on the effect of urine $\mathrm{Cd}$ (i.e., a $5 \%$ increase in magnitude) and does not improve model fit as indicated by the increase in QIC value (i.e., 452.91-444.50=+8.41). Asian race/ethnicity among female patients is a positive predictor of oocyte fertilization in Model 2 in which blood and urine $\mathrm{Cd}$ are the predictors of interest $(\mathrm{RR}=1.27, P=$ 0.0316 ), consistent with our prior report [1].

The adjusted log-binomial regression models of oocyte fertilization on urine and blood $\mathrm{Cd}$ measured in 15 male partners is also presented in Table 2. As reported previously, Model 1 , including urine $\mathrm{Cd}$ as the only predictor of interest, suggests an $81 \%$ decrease in the probability for oocyte fertilization per $\mu \mathrm{g} / \mathrm{L}$ increase in urine $\mathrm{Cd}$ concentration. In Model 2, the addition of blood Cd concentrations to Model 1 has a substantial impact on the effect of urine $\mathrm{Cd}$ (i.e., $26 \%$ increase in magnitude) and model fit is improved (i.e., 307.00-309.53=-2.53). Furthermore, Model 2 suggests that a $\mu \mathrm{g} / \mathrm{L}$ increase in blood $\mathrm{Cd}$ concentrations is associated with a $34 \%$ decrease in the probability for oocyte fertilization, conditional on urine $\mathrm{Cd}$ concentrations and other covariates. Cigarette smoking among male partners is a positive predictor of oocyte fertilization in Model 2 in which blood and urine $\mathrm{Cd}$ are the predictors of interest $(\mathrm{RR}=1.95, P<0.0001)$, consistent with our prior report [1]. In contrast to this observation, unadjusted analysis demonstrates no association between smoking and oocyte fertilization $(\mathrm{RR}=1.04, P=0.754)$ indicating that this association is confounded by the considered covariates.

\section{Discussion}

In this study we expand our prior data set [1] by incorporating blood $\mathrm{Cd}$ concentrations and present evidence suggesting the potential importance of recent male $\mathrm{Cd}$ exposure on oocyte fertilization in vitro. The results of this preliminary study raise the possibility of a gender specific effect in which blood $\mathrm{Cd}$ concentrations in male IVF partners, but not female patients, appear to be important.

The blood $\mathrm{Cd}$ concentrations measured in our samples are similar to or less than those measured for the U.S. population as reported by the National Health and Nutrition Examination Survey [9]. Median (95\% CI) blood Cd concentrations for female patients and male partners are $0.27 \mu \mathrm{g} / \mathrm{L}(0.22-0.37)$ and $0.18 \mu \mathrm{g} / \mathrm{L}(0.14-0.21)$, respectively. Median $(95 \% \mathrm{CI})$ blood $\mathrm{Cd}$ concentrations for U.S. females and males are $0.30 \mu \mathrm{g} / \mathrm{L}(<0.30(\mathrm{LOD})-0.30)$ and $0.30 \mu \mathrm{g} / \mathrm{L}(0.30-0.40)$ respectively. We previously reported a similar pattern for urine $\mathrm{Cd}$ concentrations in which the median value for female

Table 1 Distribution of blood and urine cadmium concentrations measured on the day of oocyte collection in female patients and their male partners; the Study of Metals and Assisted Reproductive Technologies (SMART)

\begin{tabular}{lcccccc}
\hline & $\mathrm{n}$ & Mean & SD & Min & Median & $\mathrm{rax}^{\mathrm{a}}$ \\
\hline Female patients & & & & & & \\
$\quad$ Blood cadmium $(\mu \mathrm{g} / \mathrm{L})$ & 50 & 0.40 & 0.51 & 0.08 & 0.27 & 3.60 \\
$\quad$ Urine cadmium $(\mu \mathrm{g} / \mathrm{g}$ creatinine) & 55 & 0.30 & 0.17 & 0.04 & 0.30 & 0.98 \\
Male partners & & & & & 0.18 & 0.97 \\
$\quad$ Blood cadmium $(\mu \mathrm{g} / \mathrm{L})$ & 33 & 0.23 & 0.19 & 0.05 & 0.17 \\
Urine cadmium $(\mu \mathrm{g} / \mathrm{g}$ creatinine) & 36 & 0.15 & 0.11 & -0.07 & 0.13 & 0.51 \\
\hline
\end{tabular}

${ }^{\text {a }}$ Correlation coefficients for blood cadmium and urine cadmium

Max, maximum value; Min, minimum value; $\mathrm{SD}$, standard deviation ${ }^{* *} P \leq 0.10$ 
Table 2 Multiple log-binomial regression models of oocyte fertilization in vitro on concentrations of urine and/or blood cadmium measured in 25 female patients and 15 male partners, with generalized estimating equations used to generate robust standard errors; the Study of Metals and Assisted Reproductive Technologies (SMART)

\begin{tabular}{|c|c|c|c|c|c|c|c|}
\hline & \multirow[t]{2}{*}{ Models ${ }^{\text {a }}$} & \multirow[t]{2}{*}{ Variables } & \multirow[t]{2}{*}{$\mathrm{RR}$} & \multicolumn{2}{|c|}{$95 \% \mathrm{CI}$} & \multirow[t]{2}{*}{$p$-value } & \multirow[t]{2}{*}{ QIC } \\
\hline & & & & Low & High & & \\
\hline \multirow[t]{3}{*}{ Female patients } & Model 1 & Urine cadmium $(\mu \mathrm{g} / \mathrm{L})$ & 1.41 & 0.62 & 3.17 & 0.412 & 444.50 \\
\hline & Model 2 & Urine cadmium $(\mu \mathrm{g} / \mathrm{L})$ & 1.48 & 0.60 & 3.66 & 0.391 & 452.91 \\
\hline & & Blood cadmium $(\mu \mathrm{g} / \mathrm{L})$ & 0.88 & 0.28 & 2.80 & 0.828 & \\
\hline \multirow[t]{3}{*}{ Male partners } & Model 1 & Urine cadmium $(\mu \mathrm{g} / \mathrm{L})$ & 0.19 & 0.03 & 1.35 & 0.097 & 309.53 \\
\hline & Model 2 & Urine cadmium $(\mu \mathrm{g} / \mathrm{L})$ & 0.14 & 0.02 & 1.32 & 0.087 & 307.00 \\
\hline & & Blood cadmium $(\mu \mathrm{g} / \mathrm{L})$ & 0.66 & 0.38 & 1.15 & 0.143 & \\
\hline
\end{tabular}

$C I$ confidence interval, $R R$ Relative risk for oocyte fertilization, $Q I C$ Quasi-likelihood Information Criteria

${ }^{a}$ Models include blood lead, blood mercury, age, race/ethnicity, cigarette smoking and urine creatinine $(\mathrm{mg} / \mathrm{dL})$ entered as covariates

IVF patients is similar to that reported for U.S. females, and the median value for the male partners is somewhat lower than that reported for U.S. males [1].

Here we report a positive correlation between urine and blood $\mathrm{Cd}$ concentrations in female patients $(r=0.47, P=$ 0.0008). This result is consistent with that from the logbinomial regression model (i.e., "Model 2"), indicating that blood $\mathrm{Cd}$ and urine $\mathrm{Cd}$ may be markers for the same exposure, vis a vis, blood $\mathrm{Cd}$ does not contribute different information than does urine $\mathrm{Cd}$. Other investigators have previously reported similar findings $(r=0.39, P<0.01)$ for the correlation between blood and urine $\mathrm{Cd}$ in women [10]. A recent study of women undergoing IVF reported no association between blood $\mathrm{Cd}$ and oocyte fertilization [11], consistent with the results of this study.

In contrast to the results for female patients, blood and urine $\mathrm{Cd}$ in males partners were not correlated $(r=0.17, P=$ 0.350 ). This result underscores the improvement detected for the fit of the log-binomial model for oocyte fertilization, upon the inclusion of blood $\mathrm{Cd}$ as an additional predictor variable (i.e., change in $\mathrm{QIC}=-2.53$ ). Numerous studies indicate inverse associations between blood $\mathrm{Cd}$ concentrations and sperm quality, including count, morphology, and motility $[12,13]$. In contrast, data describing associations between blood Cd levels and oocyte fertilization is comparatively sparse. Additional studies describe inverse association between seminal $\mathrm{Cd}$ concentrations and human fertility $[14,15]$ although we did not have these measures available.

It is tempting to speculate with regard to biologic mechanisms that might be involved in the suggested positive or inverse associations between oocyte fertilization and $\mathrm{Cd}$ exposure in female IVF patients and $\mathrm{Cd}$ exposure in male IVF partners, respectively. Several toxic metals, including $\mathrm{Cd}^{2+}$, enter eukaryotic cells directly [16] via $\mathrm{Ca}^{2+}$ channels and may directly bind to and interfere with cytoskeletal function [17] and/or the estrogen receptor [18].
Cadmium accumulating in reproductive tissues might directly enter cells of the cumulus-oocyte-complex [19, 20] or be delivered to the oocyte while bound to penetrating sperm $[14,21]$. Once within a cell $\mathrm{Cd}^{2+}$ might elicit oxidative damage directly [22], leading to mitotic failure or cell death, or indirectly by depleting intracellular stores of thiols and thereby limiting anti-oxidant enzyme activity [23]. Furthermore, $\mathrm{Cd}^{2+}$ may bind the intracellular estrogen receptor $[18,24]$ in agonistic fashion and might thereby alter the complex gonadotropin-mediated process of fertilization induced meiotic resumption [25].

A penetrating sperm not only delivers the paternal haploid genome to an oocyte but also a heretofore unknown meiosis resumption signal and a centriole which is required for microtubule assembly and initial zygote development [26]. Metals bound to the cytoskeletal elements of spermatozoa, such as $\mathrm{Cd}$, might interfere with resumption of oocyte meiosis and consequently oocyte fertilization in ICSI and conventional insemination cases [27]. Alternately, Cd-induced toxicity of sperm may directly account for the inverse association suggested for male urine $\mathrm{Cd}$ and oocyte fertilization in this study [14]. Unfortunately, we did not have sperm quality/function data available for this preliminary study and thus evaluation of this possibility will also require further study. The conditionally-independent effects suggested for recent and long-term $\mathrm{Cd}$ exposure among males may reflect different biologic mechanisms associated with the distinct exposure patterns, such as oxidative stress and estrogen receptor binding, respectively.

To our knowledge, this preliminary study constitutes the first report of a possible association between oocyte fertilization in vitro and recent $\mathrm{Cd}$ exposure among males, conditionally independent of the effects of long-term exposure. Due to the limited sample size we were unable to simultaneously evaluate exposures in women and men, or to explore potential effect modification by insemination 
protocol, a scenario in which associations between metals and oocyte fertilization may differ in ICSI vs. conventional insemination couples. As an intermediate variable in the causal pathway between exposure and endpoint, insemination protocol does not confound associations between metals and oocyte fertilization; however, differences in the proportions of fertilized oocytes by these approaches raise the possibility for effect modification. Furthermore, a substantial proportion of values were below the LOD which somewhat undermines these study results. The results of this study can only be considered preliminary; however, they indicate that both recent and long-term $\mathrm{Cd}$ exposure should be considered when assessing the impact of exposure on oocyte fertilization during IVF.

\section{Conclusion}

A future study, with a larger sample size will permit not only increased statistical power but will facilitate simultaneous consideration of female and male exposures [28], both long-term and recent, and oocyte fertilization during IVF, and permit consideration of effect modification by insemination protocol.

Acknowledgements We would like to thank Chris D. Palmer, $\mathrm{Ph}$. $\mathrm{D}$, for providing expert input regarding determination of metals in blood specimens and to extend our gratitude to the study participants whose generosity made this study possible. This work was not supported by any external grant funding. Institutional discretionary research funds available to Drs. Bloom and Fujimoto were used to support this work.

Open Access This article is distributed under the terms of the Creative Commons Attribution Noncommercial License which permits any noncommercial use, distribution, and reproduction in any medium, provided the original author(s) and source are credited.

\section{References}

1. Bloom MS, Parsons PJ, Steuerwald AJ, Schisterman EF, Browne $\mathrm{RW}$, Kim K, et al. Toxic trace metals and human oocytes during in vitro fertilization (IVF). Reprod Toxicol 2010;29:298-305

2. Agency for Toxic Substances and Disease Registry (ATSDR). Toxicological profile for cadmium. Atlanta, GA 1999

3. Lauwerys R, Bernard A, Roels H, Buchet J. Cadmium: exposure markers as predictors of nephrotoxic effects. Clin Chem. 1994;40:1391-4.

4. Palmer CD, Lewis Jr ME, Geraghty CM, Barbosa Jr F, Parsons PJ. Determination of lead, cadmium and mercury in blood for assessment of environmental exposure: a comparison between inductively coupled plasma-mass spectrometry and atomic absorption spectrometry. Spectrochim Acta Part B Atom Spectrosc. 2006;61:980-90.

5. Schisterman EF, Vexler A, Whitcomb BW, Liu A. The limitations due to exposure detection limits for regression models. Am J Epidemiol. 2006;163:374-83.
6. Greenland S, Pearl J, Robins JM. Causal diagrams for epidemiologic research. Epidemiology. 1999;10:37-48.

7. Hernan MA, Hernandez-Diaz S, Robins JM. A structural approach to selection bias. Epidemiology. 2004;15:615-25.

8. Pan W. Akaike's information criterion in generalized estimating equations. Biometrics. 2001;57:120-5.

9. U.S. Centers for Disease Control and Prevention (CDC). Third national report on human exposure to environmental chemicals. Atlanta, GA 2005

10. Shimbo S, Zhang ZW, Moon CS, Watanabe T, Nakatsuka H, Matsuda-Inoguchi N, et al. Correlation between urine and blood concentrations, and dietary intake of cadmium and lead among women in the general population of Japan. Int Arch Occup Environ Health. 2000;73:163-70.

11. Al-Saleh I, Coskun S, Mashhour A, Shinwari N, El-Doush I, Billedo G, et al. Exposure to heavy metals (lead, cadmium and mercury) and its effect on the outcome of in-vitro fertilization treatment. Int J Hyg Environ Health. 2008;211:560-79.

12. Inhorn MC, King L, Nriagu JO, Kobeissi L, Hammoud N, Awwad $\mathrm{J}$, et al. Occupational and environmental exposures to heavy metals: risk factors for male infertility in Lebanon? Reprod Toxicol. 2008;25:203-12.

13. Meeker JD, Rossano MG, Protas B, Diamond MP, Puscheck E, Daly D, et al. Cadmium, lead, and other metals in relation to semen quality: human evidence for molybdenum as a male reproductive toxicant. Environ Health Perspect. 2008;116:14739.

14. Benoff S, Hauser R, Marmar JL, Hurley IR, Napolitano B, Centola GM. Cadmium concentrations in blood and seminal plasma: correlations with sperm number and motility in three male populations (infertility patients, artificial insemination donors, and unselected volunteers). Mol Med. 2009;15:248-62.

15. Seren G, Kaplan M, Ibar H. A comparative study of human seminal plasma and blood serum trace elements in fertile and infertile men. Anal Lett. 2002;35:1785-94.

16. Foulkes EC. Transport of toxic heavy metals across cell membranes. Proc Soc Exp Biol Med. 2000;223:234-40.

17. DalleDonne I, Milzani A, Colombo R. Actin assembly by cadmium ions. Biochim Biophys Acta Mol Cell Res. 1997;1357:517.

18. Johnson MD, Kenney N, Stoica A, Hilakivi-Clarke L, Singh B, Chepko G, et al. Cadmium mimics the in vivo effects of estrogen in the uterus and mammary gland. Nat Med. 2003; 9:1081-4.

19. Younglai EV, Foster WG, Hughes EG, Trim K, Jarrell JF. Levels of environmental contaminants in human follicular fluid, serum, and seminal plasma of couples undergoing in vitro fertilization. Arch Environ Contam Toxicol. 2002;43:121-6.

20. Zenzes MT, Krishnan S, Krishnan B, Zhang H, Casper RF. Cadmium accumulation in follicular fluid of women in in vitro fertilization-embryo transfer is higher in smokers. Fertil Steril. 1995;64:599-603.

21. Yanagimachi R. Intracytoplasmic sperm injection experiments using the mouse as a model. Hum Reprod. 1998;13:87-98.

22. Lee DH, Lim JS, Song K, Boo Y, Jacobs Jr DR. Graded associations of blood lead and urinary cadmium concentrations with oxidative-stress-related markers in the U.S. population: results from the third National Health and Nutrition Examination Survey. Environ Health Perspect. 2006;114:350-4.

23. Ercal N, Gurer-Orhan H, Aykin-Burns N. Toxic metals and oxidative stress part I: mechanisms involved in metal-induced oxidative damage. Curr Top Med Chem. 2001;1:529-39.

24. Garcia-Morales P, Saceda M, Kenney N, Kim N, Salomon DS, Gottardis MM, et al. Effect of cadmium on estrogen receptor levels and estrogen-induced responses in human breast cancer cells. J Biol Chem. 1994;269:16896-901. 
25. Zhang M, Ouyang H, Xia G. The signal pathway of gonadotrophins-induced mammalian oocyte meiotic resumption. Mol Hum Reprod. 2009;15:399-409.

26. Barroso G, Valdespin C, Vega E, Kershenovich R, Avila R, Avendaño C, et al. Developmental sperm contributions: fertilization and beyond. Fertil Steril. 2009;92:835-48.
27. Rawe VY, Diaz ES, Abdelmassih R, Wojcik C, Morales P, Sutovsky $\mathrm{P}$, et al. The role of sperm proteasomes during sperm aster formation and early zygote development: implications for fertilization failure in humans. Hum Reprod. 2008;23:573-80.

28. Joffe M. Infertility and environmental pollutants. Br Med Bull. 2003;68:47-70. 